\title{
HDAC4 and HDAC7 Promote Breast and Ovarian Cancer Cell Migration by Regulating Emt Relative Marker
}

\author{
Shuangping Liư ${ }^{1}$, Hao Sheng ${ }^{2}$, Jiao Liu², Changliang Shan ${ }^{3 *}$ and Shuai Zhang** \\ ${ }^{1}$ Department of Pathology, Medical School,Dalian University, Dalian, Liaoning 116622, China. \\ ${ }^{2}$ Biomedical Translational Research Institute, Jinan University, Guangzhou, Guangdong 510632, China. \\ ${ }^{3}$ College of Pharmacy and Tianjin Key Laboratory of Molecular Drug Research, Nankai University, Tianjin 300350, China. \\ ${ }^{4}$ School of Integrative Medicine, Tianjin University of Traditional Chinese Medicine, Tianjin 300000, China.
}

*Corresponding author: Shuai Zhang, School of Integrative Medicine, Tianjin University of Traditional Chinese Medicine, Tianjin 300000, China, Email: shuaizhang@tjutcm.edu.cn

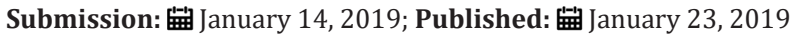

\begin{abstract}
Breast and ovarian cancer have been remained as a highly malignant tumor among women, posing a serious threat to women health worldwide. In this study, we were aimed to investigate the underlying mechanism of breast and ovarian cancer cell migration. Wound healing assay showed that MDA-MB-231 and C13* have higher migration potential compare with MCF-7 and OV2078 cells, as well as regulated epithelial-mesenchymal transition (EMT) marker. We found that HDAC4 and HADC7 mRNA are up regulated in MDA-MB-231 and C13* cells. Moreover, target HDAC4 and HDAC7 by TSA or shRNA block MDA-MB-231and C13* migration. These results reveal a new link between HDACs and EMT in the regulation of breast and ovarian cancer migration.
\end{abstract}

Keywords: Histone deacetylases (HDACs); Epithelial-mesenchymal transition (EMT); Migration

\section{Introduction}

Breast and ovarian cancer have been remained as a highly malignant tumor among women, posing a serious threat to women health worldwide [1-3]. In 2012, Breast cancer was considered as the fifth leading of cancer death, having caused 1.67 million new cases which accounted for $25 \%$ of all new cancer cases in the world [4]. Ovarian cancer is the fifth most common cause of cancer deaths in women and the tenth most common cancer among women in the United States, and the leading cause of death from gynecologic cancer [5]. They have become the leading cause of cancer death among females. In recent years, significant progress has been achieved in surgical techniques and chemotherapy regimens. However, relapse remains almost inevitable in patients with advanced breast and ovarian cancer [6]. It is thus important to understand the details of the underlying molecular mechanism of cancer pathogenesis that promote cancer migration, as this may provide both new imaging and therapeutic targets.

The role of epigenetic alterations, including the acetylation status of histones and DNA methylation, has been an important focus in studies on the development of human cancers, and such changes are often an early event in tumorigenesis [7,8]. Histone deacetylase (HDAC) family members remove acetyl groups from the lysine residues of histones, leading to controlling gene expression, which is involved in many diseases, including age-related tissue degeneration, heart failure and cancer [9]. The specific interactions of various HDACs and their effects on many types of cancer were clarified. HDACs are often highly expressed in various types of cancer and promote cancer progress, so they are attractive anticancer targets [10-14]. HDAC activity in human tumors leads to conformational changes within the nucleosome, which results in the transcriptional repression of genes during migration and metastasis [15-17]. Changes in HDAC expression levels have been associated with clinical prognosis in patients with invasive cancer, including breast cancer [18]. In this study, we aim to shed light on the mechanisms of breast and ovarian cancer metastasis. We show that MDA-MB-231and C13* have higher potently migration than MCF-7 and OV2078. And increase EMT marker and HDAC4/7 expression. Inhibition of HDAC4/7 can block the EMT marker express of MDAMB-231and C13*, leading to migration arrest. These results support the hypothesis that cancer cells have higher HDACs promote cancer migration through regulating (EMT) marker express. Thus, by developing HDAC isoforms as potential biomarkers for breast and ovarian cancer metastasis, the present study can be extended to developing therapies for breast cancer invasion. 


\section{Materials and Methods}

\section{Cell culture}

Human ovarian surface epithelial cancer cells OV2008 and C13* were gifts from Dr. Benjamin K. Tsang (University of Ottawa, Ontario, Canada). OV2008 and C13* cells were cultured in RPMI 1640 medium with $10 \%$ fetal bovine serum (FBS) [19]. MCF-7 and MDA-MB-231cells were cultured in Dulbecco Modified Eagle Medium (DMEM) with 10\% FBS.

\section{Quantitative real-time PCR}

Total cellular RNA was extracted using the EastepR Super RNA Extract reagent Kit (Promega). cDNA was generated from purified RNA using Prime Scipt TM RT reagent Kit (Takara) according to the manufacturer's instructions. Quantitative Real-time PCR (qRT-PCR) was performed using SYBR Green (Biotool) on a Bio-rad CFX96 Real-time PCR System (Bio-rad). Relative target mRNA (primer in the Supplementary Table) levels were normalized to $\beta$-actin expression

\section{Wound healing assay}

Cells were grown in 6-well to $80 \%$ confluency. Scratches were made along the center of the dish with $200 \mu$ lips. Cells were subsequently incubated in $\mathrm{CO}_{2}$ chamber and images were captured at regular intervals. Images were analyzed by Image J software. Distance between two approaching growth fronts at any time was measured and its ratio with that at 0 hour was represented as the percentage of cell migration.

\section{Statistical analysis}

Statistical analyses were performed using Student's ttest. All data obtained from three independent experiments performed in triplicate and are presented as the mean \pm standard error. $\mathrm{P}<0.05$ was considered to indicate a statistically significant difference.

\section{Results}

\section{Higher migration of breast and ovarian cancer cells have higher EMT marker express}

We first compared the migration between MDA-MB-231/C13* and MCF-7 /OV2078 cells, wound healing assay showed that MDAMB-231and C13* have higher potential migration than MCF-7 and OV2078 cells. We next examined the expression of EMT marker in MDA-MB-231/C13* and MCF-7 /OV2078 cells, real time PCR results show that E-cadherin was decreased in MDA-MB-231/ C13*. N-cadherin and vimentin were increased in MDA-MB-231/ C13*. Slug was increased in MDA-MB-231 compared with MCF-7, but not difference between C13* and OV2008. Sanil was increased in MDA-MB-231 compare with MCF-7, but it was decrease in $\mathrm{C} 13^{*}$ compared with OV2008. Together, these results show that MDAMB-231and C13* have higher migration potential rate than MCF-7 and OV2078 cells, as well as regulated most EMT marker (Figure 1).

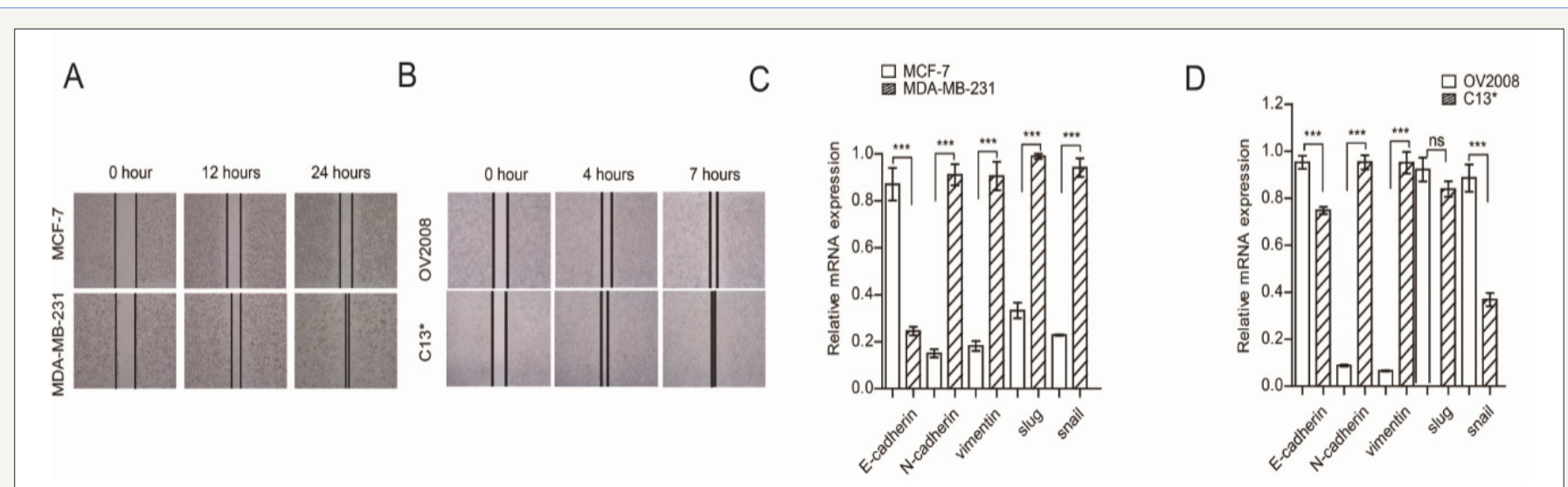

Figure 1: Higher migration of breast and ovarian cancer cells have higher EMT marker express.

(A) Wound healing assay check migration of MDA-MB-231 and MCF-7.

(B) Wound healing assay check migration of OV2008 and C13*.

(C) Real time PCR checks EMT marker express in MDA-MB-231 and MCF-7.

(D) Real time PCR checks EMT marker express in OV2008 and C13*. Error bars represent mean values \pm SD from three replicates of each sample $\left({ }^{*} \mathrm{P}<0.05 ;{ }^{* *} \mathrm{P}<0.01 ;{ }^{* * *} \mathrm{P}<0.001\right)$.

\section{HDAC4 and HDAC7 expression are significantly higher in MDA-MB-231and C13*}

Several lines of evidence suggest that cancer cells regulate the HDACs to support cancer cell migration. However, the role of HDACs in cancer cell migration have not been clearly described. We want to know whether HDACs involve in promoting cell migration. So, we compared the HDACs expression between MDA-MB-231/C13* and MCF-7 /OV2078 cells. Our results showed that HDAC4 and HDAC7 were upregulated in both MDA-MB-231 and C13* cells. Taken together, these results suggest that higher migration cells may regulate HDAC4 and HDAC7 expression as a mechanism for promoting cell migration (Figure 2). 


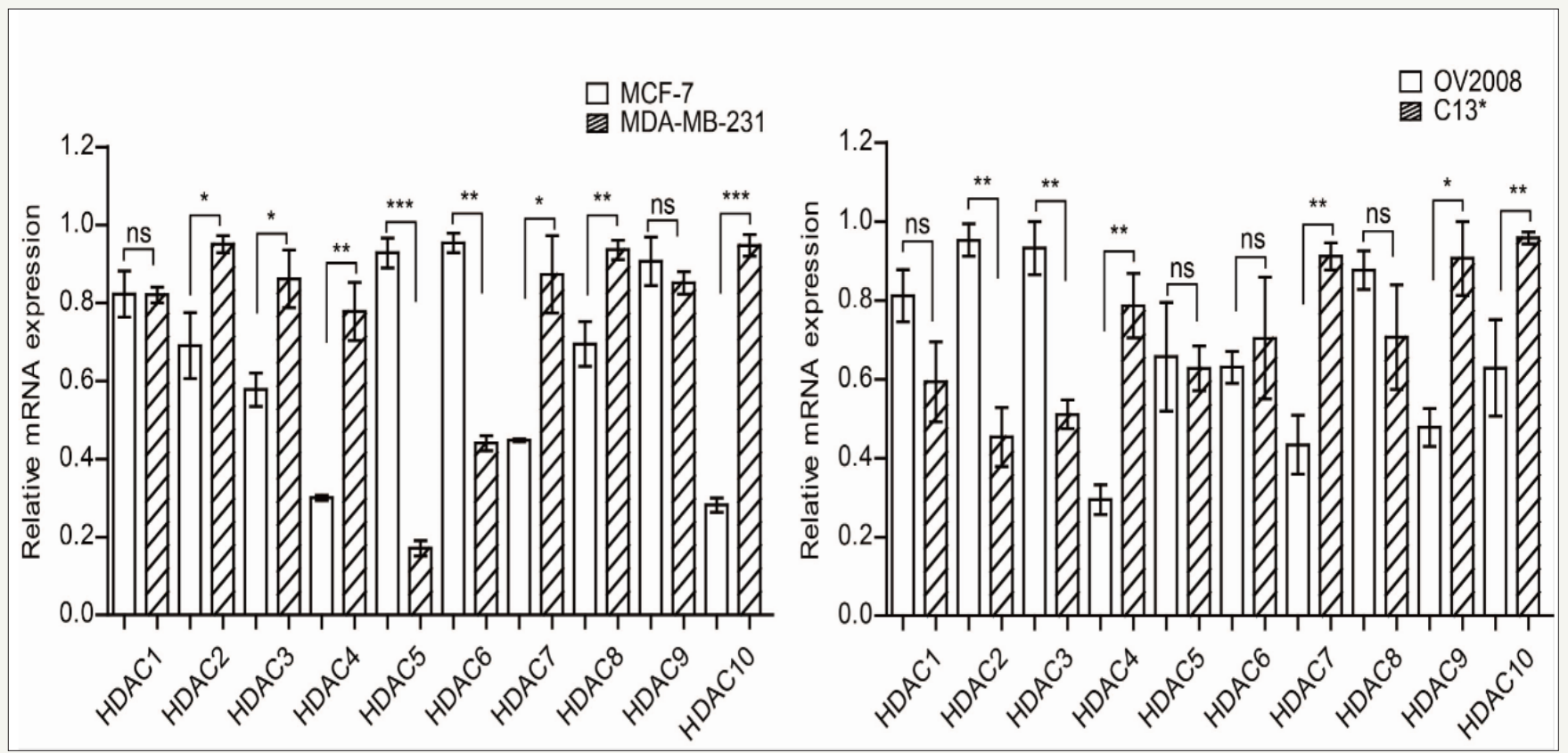

Figure 2: HDAC4 and HDAC7 expression were upregulated in MDA-MB-231/C13* compare with MCF-7/OV2008.

(A) Real time PCR checks EMT marker express in MDA-MB-231 and MCF-7.

(B) Real time PCR checks EMT marker express in OV2008 and C13*. Error bars represent mean values \pm SD from three replicates of each sample $\left({ }^{*} \mathrm{P}<0.05 ;{ }^{* *} \mathrm{P}<0.01 ;{ }^{* * *} \mathrm{P}<0.001\right)$.

Targeting HDAC4 and HDAC7 by HDACs inhibitor Trichostatin A (TSA) decreased cell migration and regulating EMT marker expression

To validate our hypothesis, we analyzed cell migration in MDAMB-231 and C13* cells when treated with Trichostatin A (TSA), the HDACs inhibitor. Wound healing assay showed that TSA significantly inhibited MDA-MB-231and C13* cell migration. We next examined the expression of EMT marker in MDA-MB-231, C13* cells when treated with TSA, real time PCR results show that E-cadherin was increased in MDA-MB-231/C13*. N-cadherin, vimentin and snail were decreased, and Slug was not changed in MDA-MB-231 when treated with TSA. N-cadherin, vimentin and snail were increased except Slug in C13* when treated with TSA. Together, these results show that MDA-MB-231and C13* cell migration were blocked by treated with TSA (Figure 3).
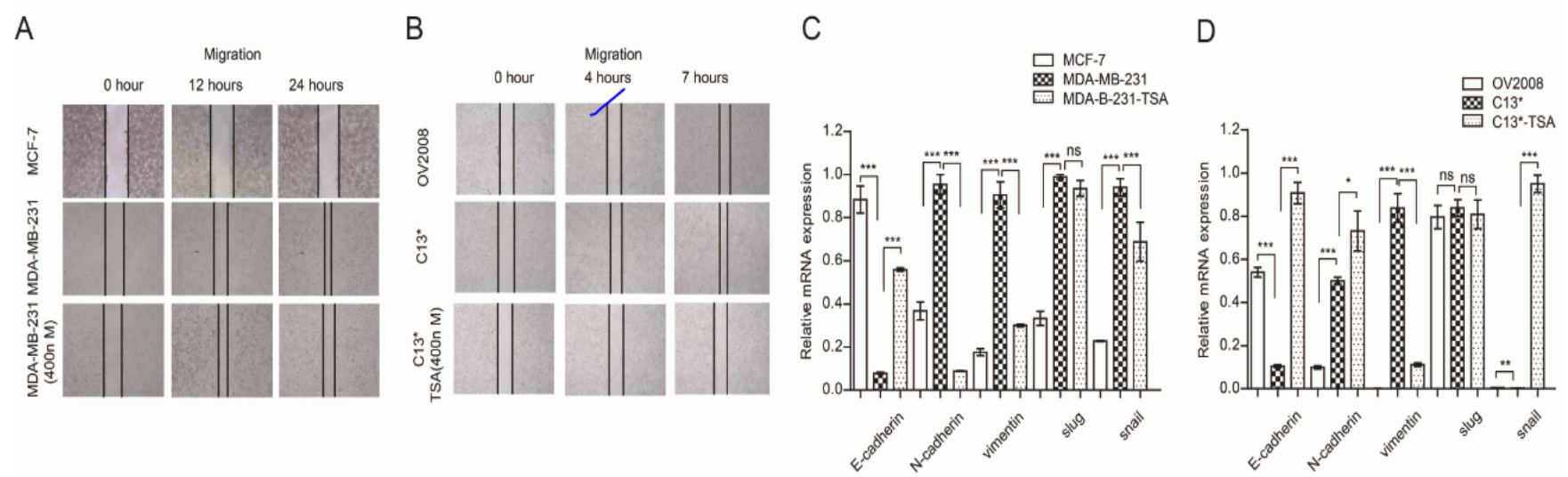

Figure 3: Targeting HDAC4 and HDAC7 by HDACs inhibitor Trichostatin A (TSA) decreased cell migration and regulating EMT marker expression.

(A) Wound healing assay check migration of MDA-MB-231 and MCF-7 when treated with TSA.

(B) Wound healing assay check migration of OV2008 and C13* when treated with TSA.

(C) Real time PCR checks EMT marker express in MDA-MB-231 and MCF-7 when treated with TSA.

(D) Real time PCR check EMT marker express in OV2008 and C13* when treated with TSA. Error bars represent mean values \pm SD from three replicates of each sample ( ${ }^{*} \mathrm{P}<0.05 ;{ }^{*} \mathrm{P}<0.01$; $\left.{ }^{* * *} \mathrm{P}<0.001\right)$.

How to cite this article: Shuangping L, Hao S, Jiao L, Changliang S, Shuai Z. HDAC4 and HDAC7 Promote Breast and Ovarian Cancer Cell Migration by Regulating Emt Relative Marker. Nov Appro in Can Study. 2(1). NACS.000535.2019. DOI: 10.31031/NACS.2019.02.000535 
Targeting HDAC4 and HDAC7 by HDACs shRNA decreased cell migration and regulating EMT marker expression

To further confirm our founding, wound healing assay showed that targeting HDAC4 and HDAC7 by their shRNA significantly inhibited MDA-MB-231and C13* cell migration. We next examined the expression of EMT marker in MDA-MB-231, C13* cells when treated with HDAC4 and HDAC7shRNA, real time PCR results show that E-cadherin and slug were increased in MDA-MB-231/C13*. $\mathrm{N}$-cadherin, vimentin and snail were decreased in MDA-MB-231/ C13* when treated with HDAC4 and HDAC7shRNA.Together, these results show that MDA-MB-231and $\mathrm{C} 13^{*}$ cell migration were blocked by suppressing HDAC4 and HDAC7 expression (Figure 4).

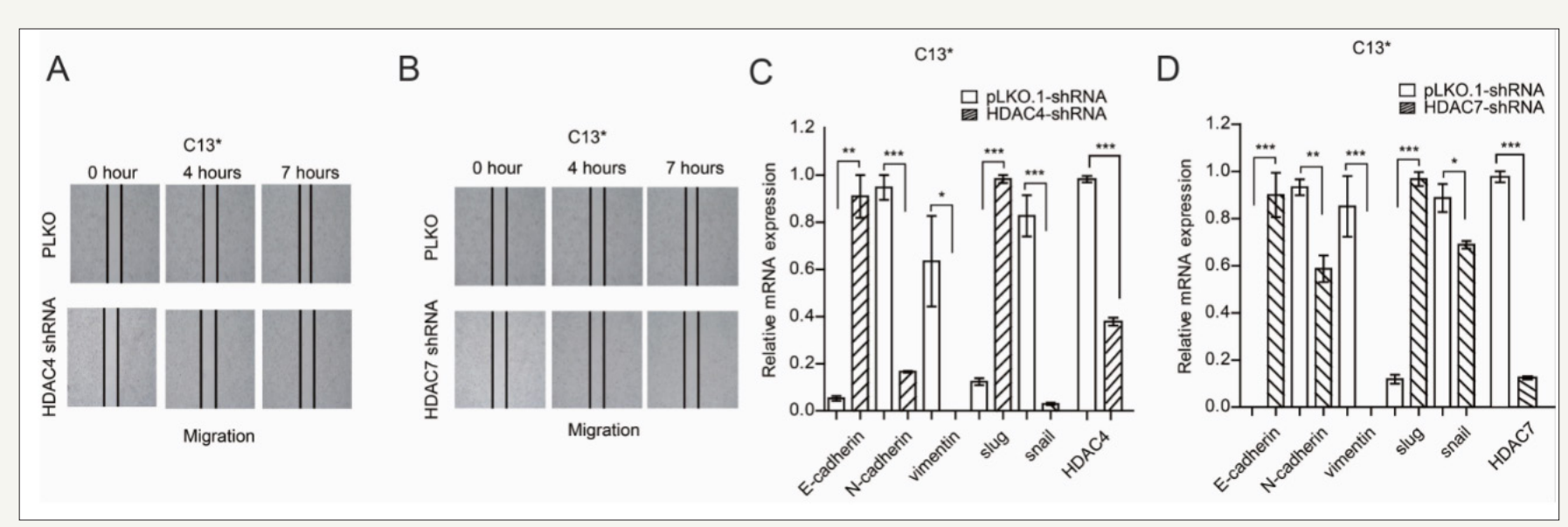

Figure 4: Targeting HDAC4 and HDAC7 by HDACs shRNA decreased cell migration and regulating EMT marker expression.

(A) Wound healing assay check migration of $\mathrm{C} 13^{*}$ when treated with HDAC4 shRNA.

(B) Wound healing assay check migration of $\mathrm{C} 13^{*}$ when treated with HDAC7 shRNA.

(C) Real time PCR check EMT marker express in $\mathrm{C} 13^{*}$ when treated with HDAC4 shRNA.

(D) Real time PCR check EMT marker express in C13* when treated with HDAC7 shRNA. Error bars represent mean values \pm SD

from three replicates of each sample $\left({ }^{*} \mathrm{P}<0.05 ;{ }^{* *} \mathrm{P}<0.01\right.$; $\left.{ }^{* *} \mathrm{P}<0.001\right)$.

\section{Discussion}

In recent years, great achievements in our understanding of breast and ovarian cancer pathogenesis have contributed to improved surgical management and chemotherapy treatment. However, the limited efficacy of novel therapeutics has become a major obstacle due to our inadequate comprehension of tumor cell metastasis. It is urgent to investigate the further molecular mechanisms underlying breast and ovarian cancer metastasis. In the present study, we demonstrated that HDAC4 and HDAC7 promoted breast and ovarian cancer cell migration via EMT-related key regulators. Metastasis is one of the most common features of malignant tumors, which is responsible for most cancer-related deaths [20]. Epithelial-to-mesenchymal transition (EMT) is a major process in cancer cell invasion and metastasis. Emerging studies have demonstrated aberrant expression of Classical (Class I,II,IV) HDACs has been linked to cancer cell metastasis [17]. These findings indicate the therapeutic potential of inhibition of Class I HDACs in targeting EMT and metastasis of cancer cells. However, the level of HDACs expression in breast and ovarian cancer and their association with the clinical outcome of the Metastasis is unclear.

In our study, we demonstrated that HDAC4 and HADC7 mRNA are overexpressed in MDA-MB-231 and C13* cells, which have higher migration potential than MCF-7 and 0V2008 cells.
Downregulation of HDAC4 and HDAC7 significantly reduced the migratory activity in DA-MB-231 and C13* cells. Moreover, epithelial-mesenchymal transition (EMT) marker were identified as a functional target of HDAC4 and HDAC7. Many studies report that EMT marker play diverse roles in the pathogenesis, progression, invasion, and metastasis in diverse human cancers. The expressions of EMT marker are frequently dysregulated in malignancies, and are associated with tumors aggressive behavior $[6,21]$. EMT has been recognized as a critical procedure regulating the pathogenesis of breast cancer [22]. Many signaling pathways are involved in the EMT program, and a theme that has emerged is that signals from soluble extracellular factors are integrated with contextual molecules for cellular phenotype regulation [23]. In conclusion, our study demonstrates that HDAC4 and HDAC7 functions as a migration promoter and has a vital role in the processes of breast and ovarian cancer cell migration. Mechanically, it exerts its function by specifically promotes the expression of EMT progression. These findings imply that HDAC4 and HDAC7 could serve as a novel therapeutic target for breast and ovarian cancer therapy.

\section{Acknowledgement}

This work was supported by the Science and Technology Program of Guangzhou (Grant 201807010003), the Program from 
the Science and Technology Department of Guangdong Province of China (Grant 2017A030313890), and the National Nature Science Foundation of China (31560312, 81672781 and 81702746). This work was also supported by the China Postdoctoral Science Foundation (2017M612839), the Fundamental Research Funds for the Central Universities (21616323, 21617433), and the Program of Introducing Talents of Discipline to Universities (111 Project, No. B16021).

\section{References}

1. Pan H, Wu N, Huang Y, Li Q Liu C, et al. (2015) Aldehyde dehydrogenase 1 expression correlates with the invasion of breast cancer. Diagn Pathol 10: 66 .

2. Taheripanah R, Balash F, Anbiaee R, Mahmoodi M, Akbari SA (2018) Breast cancer and ovulation induction treatments. Clin Breast Cancer 18(5): 395-399.

3. Zhang M, Tian Z, Sun Y (2017) Successful treatment of ovarian cancer with apatinib combined with chemotherapy: a case report. Medicine (Baltimore) 96(45): e8570.

4. McGuire S (2016) World Cancer Report 2014. Geneva, Switzerland: World Health Organization, International Agency for Research on Cancer, WHO Press, 2015. Adv Nutr 7(2): 418-419.

5. Grossman DC, Curry SJ, Owens DK, Barry MJ, Davidson KW, et al. (2018) Screening for ovarian cancer: us preventive services task force recommendation statement. JAMA 319(6): 588-594.

6. Zhao H, Kang X, Xia X, Wo L, Gu X, et al. (2016) MiR-145 suppresses breast cancer cell migration by targeting FSCN-1 and inhibiting epithelialmesenchymal transition, Am J Transl Res 8(7): 3106-3114.

7. Giacinti L, X Claudio VV, Lopez M, Giordano A (2006) Epigenetic information and estrogen receptor alpha expression in breast cancer Oncologist 11(1): 1-8.

8. Muller BM, Jana L, Kasajima A, Lehmann A, Prinzler J, et al. (2013) Differential expression of histone deacetylases HDAC1, 2 and 3 in human breast cancer--overexpression of HDAC2 and HDAC3 is associated with clinicopathological indicators of disease progression BMC Cancer 13: 215.

9. Li Y, Seto E (2016) HDACs and HDAC inhibitors in cancer development and therapy. Cold Spring Harb Perspect Med 6(10): a026831.

10. Mithraprabhu S, Kalff A, Chow A, Khong T, Spencer A (2014) Dysregulated class I histone deacetylases are indicators of poor prognosis in multiple myeloma. Epigenetics 9(11): 1511-1520.

11. Rettig, Koeneke E, Trippel F, Mueller WC, Burhenne J, et al. (2015) Selective inhibition of HDAC8 decreases neuroblastoma growth in vitro and in vivo and enhances retinoic acid-mediated differentiation. Cell Death Dis 6: e1657.
12. Seo J, Min SK, Park HR, Kim DH, Kwon MJ, et al. (2014) Expression of histone deacetylases HDAC1, HDAC2, HDAC3, and HDAC6 in invasive ductal carcinomas of the breast. J Breast Cancer 17(4): 323-331.

13. Sudo T, Mimori K, Nishida N, Kogo R, Iwaya T, et al. (2011) Histone deacetylase 1 expression in gastric cancer. Oncol Rep 26 (4): 777-782.

14. Weichert W, Denkert C, Noske A, Darb ES, Dietel M, et al. (2008) Expression of class I histone deacetylases indicates poor prognosis in endometrioid subtypes of ovarian and endometrial carcinomas. Neoplasia 10(9): 1021-1027.

15. Aghdassi, Sendler M, Guenther A, Mayerle J, Behn CO, et al. (2012) Recruitment of histone deacetylases HDAC1 and HDAC2 by the transcriptional repressor ZEB1 downregulates E-cadherin expression in pancreatic cancer. Gut 61(3): 439-448.

16. Tong ZT, Cai MY, Wang XG, Kong LL, Mai SJ, et al. (2012) EZH2 supports nasopharyngeal carcinoma cell aggressiveness by forming a corepressor complex with HDAC1/HDAC2 and Snail to inhibit E-cadherin. Oncogene 31(5): 583-594.

17. Von BJ, Eser S, Paul MC, Seidler B, Brandl M, et al. (2009) E-cadherin regulates metastasis of pancreatic cancer in vivo and is suppressed by a SNAIL/HDAC1/HDAC2 repressor complex. Gastroenterology 137(2): 361-371.

18. Park SY, Jun JA, Jeong KJ, Heo HJ, Sohn JS, et al. (2015) Histone deacetylases 1, 6 and 8 are critical for invasion in breast cancer. Oncol Rep 25(6): 1677-1681.

19. Zheng W, Feng Q, Liu J, Guo Y, Gao L, et al. (2017) Inhibition of 6-phosphogluconate dehydrogenase reverses cisplatin resistance in ovarian and lung cancer. Front Pharmacol 8: 421.

20. Eccles SA, Paon L (2005) Breast cancer metastasis: when, where, how? Lancet 365(9664): 1006-1007.

21.Xu S, Zhan M, Wang J (2017) Epithelial-to-mesenchymal transition in gallbladder cancer: from clinical evidence to cellular regulatory networks. Cell Death Discov 3: 17069.

22. Brown RL, Reinke LM, Damerow MS, Perez D, Chodosh LA, et al. (2011) CD44 splice isoform switching in human and mouse epithelium is essential for epithelial-mesenchymal transition and breast cancer progression. J Clin Invest 121(3): 1064-1074.

23. Acloque H, Adams MS, Fishwick K, Bronner FM, Nieto MA (2009) Epithelial-mesenchymal transitions: the importance of changing cell state in development and disease. J Clin Invest 119(6): 1438-1449.
Creative Commons Attribution 4.0 International License

For possible submissions Click Here

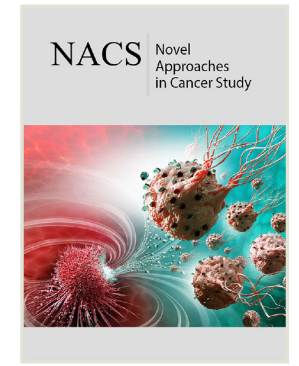

\section{Novel Approaches in Cancer Study}

\section{Benefits of Publishing with us}

- High-level peer review and editorial services

- Freely accessible online immediately upon publication

- Authors retain the copyright to their work

- Licensing it under a Creative Commons license

- Visibility through different online platforms 